\title{
Femtosecond pulsed laser deposition of indium on Si(100)
}

\author{
Mohamed A. HAFEZ and Hani E. ELSAYED-ALI
}

\begin{abstract}
Department of Electrical and Computer Engineering and the Applied Research Center, Old Dominion University, Norfolk, VA-23529
\end{abstract}

E-mail: helsayed@odu.edu

\begin{abstract}
Deposition of indium on $\mathrm{Si}(100)$ substrates is performed under ultrahigh vacuum with an amplified Ti:sapphire laser (130 fs) at wavelength of $800 \mathrm{~nm}$ and laser fluence of $0.5 \mathrm{~J} / \mathrm{cm}^{2}$. Indium films are grown at room temperature and at higher substrate temperatures with a deposition rate of $0.05 \mathrm{ML} /$ pulse. Reflection high-energy electron diffraction (RHEED) is used during the deposition to study the growth dynamics and the surface structure of the grown films. The morphology of the grown films is examined by ex situ atomic force microscopy (AFM). At room temperature indium is found to form epitaxial two-dimensional layers on the $\mathrm{Si}(100)-(2 \times 1)$ surface followed by threedimensional islands. AFM images show different indium island morphologies such as hexagonal and elongated shapes. At substrate temperatures of 400-420 ${ }^{\circ} \mathrm{C}$, RHEED intensity oscillations are observed during film growth indicating that the indium film grows in the layer-by-layer mode.

DOI: $10.2961 /$ jlmn.2006.01.0009
\end{abstract}

Keywords: femtosecond laser, indium, silicon, RHEED, 3D islands, AFM

\section{Introduction}

The growth of high quality metal films on Si surfaces in the form of coherent islands and nanoclusters is of interest to electronic devices and nanotechnology. Pulsed laser deposition (PLD) is a thin film deposition technique, which has been used to fabricate a wide range of materials [1]. PLD produces energetic species, which results in the increase of the sticking coefficients and adatom surface mobility, enhancing epitaxy [2]. Epitaxial thin films of InP on GaAs(100) and Si on Si(100) were obtained using PLD $[3,4]$. Using femtosecond (fs) laser minimizes or eliminates particulates formation, which improves the grown thin film quality. Compared to other lasers, ultrashort pulses of fs laser significantly alter the processes associated with ablation [5]. Rapid energy deposition reduces liquid phase formation and the heat-affected zone, which are usually present during PLD with nanosecond pulses. The film structural properties can be controlled by the laser parameters, such as laser fluence, pulse width, and repetition rate.

Growth of group-III metals on clean $\mathrm{Si}(100)-(2 \times 1)$ is important to the process of silicon doping in molecular beam epitaxy (MBE) and the growth of III-V and III-IV semiconductors [6-8]. It has also been observed that groupIII metals grown on Si from self-assembled wires and nanoclusters with potential applications in devices [9-11]. Knowledge of indium (In) growth modes on $\mathrm{Si}(100)-(2 \times 1)$ and development of new methods of its growth are important to various device applications and possible applications in preparation of clean and ordered Si surfaces [12].

Reflection high-energy electron diffraction (RHEED) is a useful in situ technique to study the thin film growth mode and surface morphology. The incident electron beam hits the surface at near-grazing angle, gets diffracted from the surface, and then the diffraction pattern is observed on a phosphor screen. Therefore, real time RHEED is employed to observe the surface structure, allowing us to differentiate between layer-by-layer growth and the growth of three-dimensional (3D) islands.

The RHEED study of In deposition on $\mathrm{Si}(100)-(2 \times 1)$ at room temperature (RT) by femtosecond pulsed laser deposition (fsPLD) showed that In grew in 3D islands of different surface morphology. AFM images of the In films showed regular different island shapes such as elongated, hexagonal, and hemispherical islands. The height of the islands varied from 5 to $60 \mathrm{~nm}$. When the $\mathrm{Si}(100)-(2 \times 1)$ substrate was heated to $400-420{ }^{\circ} \mathrm{C}$, In formed $(4 \times 3)$ superstructure as observed from the RHEED patterns. AFM images showed growth of $\sim 20-\mathrm{nm}$ almost spherical In nanoclusters on surface terraces.

\section{Experiment}

The In films were performed in an ultrahigh vacuum (UHV) PLD chamber. The base pressure during deposition was in the low $10^{-9}$ Torr. An amplified Ti:sapphire laser (pulse width 130 fs FWHM) operating at a wavelength of $800 \mathrm{~nm}$ and repetition rates of 1 and $2 \mathrm{~Hz}$ was used to ablate the In target. The laser was incident on the $99.99 \%$ pure In target at $\sim 45^{\circ}$. Laser radiation was focused onto the In target using a convex lens with $30 \mathrm{~cm}$ focal length. The target was rotated at a speed of $2 \mathrm{rpm}$ to minimize particulate formation. A half-waveplate and a polarizer are used to control the laser fluence for ablation. The target-tosubstrate distance was $\sim 5 \mathrm{~cm}$. RHEED is used to observe the surface of the substrate and the grown In film during deposition. The RHEED gun was operated at electron energy of $8.6 \mathrm{keV}$. The RHEED patterns were acquired by a charge-coupled device camera.

The $\sim 5 \times 10 \mathrm{~mm}^{2}$ Si substrates were cut from a Si(100) wafer (p-type, boron-doped, thickness of $500 \mu \mathrm{m})$. The Si surfaces were misoriented from the low-index (100) plane 
with miscut angle of $1^{0}$ to the $\left\langle 110> \pm 0.5^{\circ}\right.$. The $\operatorname{Si}(100)$ $(2 \times 1)$ surface was prepared by chemical etching just prior to loading in the UHV chamber followed by in situ heat cleaning to $600{ }^{\circ} \mathrm{C}$ for several hours using a direct current and then flashing at $\sim 1100{ }^{\circ} \mathrm{C}$. The films were deposited with the Si substrate kept at RT and $400-420{ }^{\circ} \mathrm{C}$. The deposition rate was estimated from RHEED oscillations and surface profiler to be $\sim 0.05 \mathrm{ML} /$ pulse.

\section{Results and discussion}

Surface preparation of the substrate, such as the $\mathrm{Si}(100)$ $(2 \times 1)$, as well as the deposition conditions are important factors in fabrication high quality metallic thin films. When In was deposited on $\mathrm{Si}(100)-(1 \times 1)$ at room temperature by fsPLD, the In films showed $(1 \times 1)$ RHEED structure at the initial stage of growth and then the pattern disappeared showing high RHEED background. Next, we deposited In films on $\mathrm{Si}(100)-(2 \times 1)$ at room temperature. Just prior to In deposition, the $\mathrm{Si}(100)-(2 \times 1)$ was raised in temperature to $\sim 1000{ }^{\circ} \mathrm{C}$ for $\sim 2$ minutes by direct current heating. The heating was then terminated and the substrate cooled down to RT. The RHEED pattern acquired after this annealing showed clean reconstructed $\mathrm{Si}(100)-(2 \times 1)$ with less RHEED background, as shown in Fig. 1(a), indicating a smooth well-ordered surface. Pulsed laser deposition of In was then started with the Si substrate kept at RT. The laser was operated at $2 \mathrm{~Hz}$ repetition rate and a fluence of 0.5 $\mathrm{J} / \mathrm{cm}^{2}$ on the In target. Figure 1(b) shows the RHEED pattern of the In film after a deposition time of $20 \mathrm{~s}$. The RHEED pattern was taken along the [011] azimuth. The initial stage of In growth was epitaxial 2D $(2 \times 1)$ structure. Using a laser repetition rate of $1 \mathrm{~Hz}$ and a laser fluence of $0.3 \mathrm{~J} / \mathrm{cm}^{2}$, the same behavior of initially observing the $(2 \times 1)$ RHEED structure during In growth was also observed. With continued In deposition, for both conditions, the integral and half-order RHEED streaks continuously decreased in intensity until the RHEED pattern became faint showing wide rings.
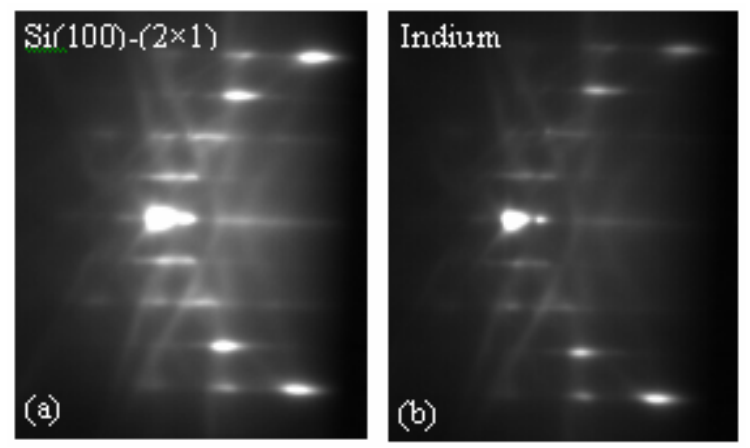

Fig. 1 (a) RHEED pattern of $\mathrm{Si}$ substrate before deposition and (b) of In film after $20 \mathrm{~s}$ of deposition at RT.

We monitored the width of the RHEED specular beam to observe the morphological changes in the $\mathrm{In} / \mathrm{Si}(100)-(2 \times 1)$ system. Figure 2(a) shows the deposition time dependence of the full width at half maximum (FWHM) taken along the RHEED specular beam in the [011] azimuthal direction. The total deposition time was 380 s. As soon as the deposition started, the FWHM dropped suddenly and then starting to decay at a slower rate with further deposition. After 70 s of deposition, the FWHM was reduced to 22\% of its original value before deposition. This indicates an increased average crystalline size and low surface roughness. However, after $230 \mathrm{~s}$ the FWHM started to increase indicating the development of surface roughness due to formation of 3D islands. RHEED pattern of the In film after terminating the deposition is shown in Fig. 2(b) and shows high background with wide faint rings indicating random in-plane crystallographic orientation.

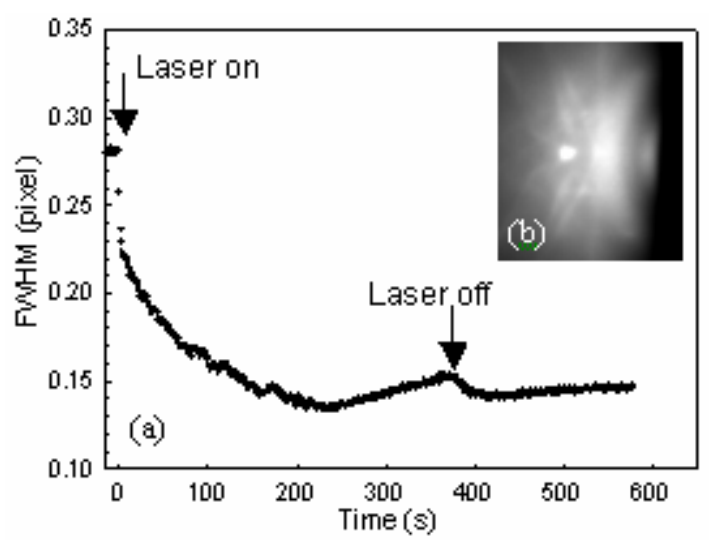

Fig. 2 (a) Deposition time dependence of the FWHM of the RHEED specular beam. (b) RHEED pattern of In film after terminating the deposition.
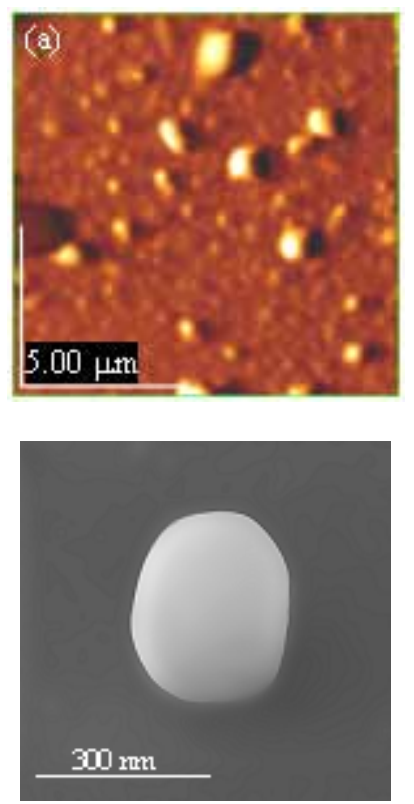

Fig. 3 (a) AFM image of In film grown on $\mathrm{Si}(100)$ (1×1) substrate. (b) SEM image of an In island.

The morphology of the grown In films were examined ex situ using the AFM. An In film was grown on $\mathrm{Si}(100)$ (1×1) substrate by fsPLD at room temperature. Figure 3(a) shows an AFM image after deposition of In on $\mathrm{Si}(100)$ (1×1) using $1 \mathrm{~Hz}$ laser repetition rate and $0.5 \mathrm{~J} / \mathrm{cm}^{2}$ laser fluence. The In islands have irregular shape and varied in size. Some islands show a faceted structure as seen in the scanning electron microscope (SEM) image in Fig. 3(b). Although, the surface of the substrate before deposition 
was not reconstructed, some crystalline islands were formed.

Next, a series of In films was grown on $\mathrm{Si}(100)-(2 \times 1)$ substrates by fsPLD via interval deposition, i.e. deposition for a given amount of time followed by an interval of no deposition. The AFM images show 3D In islands, which are found at different surface locations. The In islands took a variety of shapes such as hexagonal, elongated, and hemispherical islands. These different morphologies are related to the deposition conditions and substrate surface condition before deposition. As an example, Fig. 4(a) and (b) show 3D AFM images of elongated and hemispherical In islands grown on $\mathrm{Si}(100)-(2 \times 1)$ by fsPLD at RT. Line scans along and across an elongated island indicate an average length and width of 200 and $60 \mathrm{~nm}$, respectively. The line profile shows a height of $\sim 4.5 \mathrm{~nm}$. The hemispherical islands have a smaller size compared to the other In island types. The line scan shows an average size of $50 \mathrm{~nm}$ and height of $6 \mathrm{~nm}$.
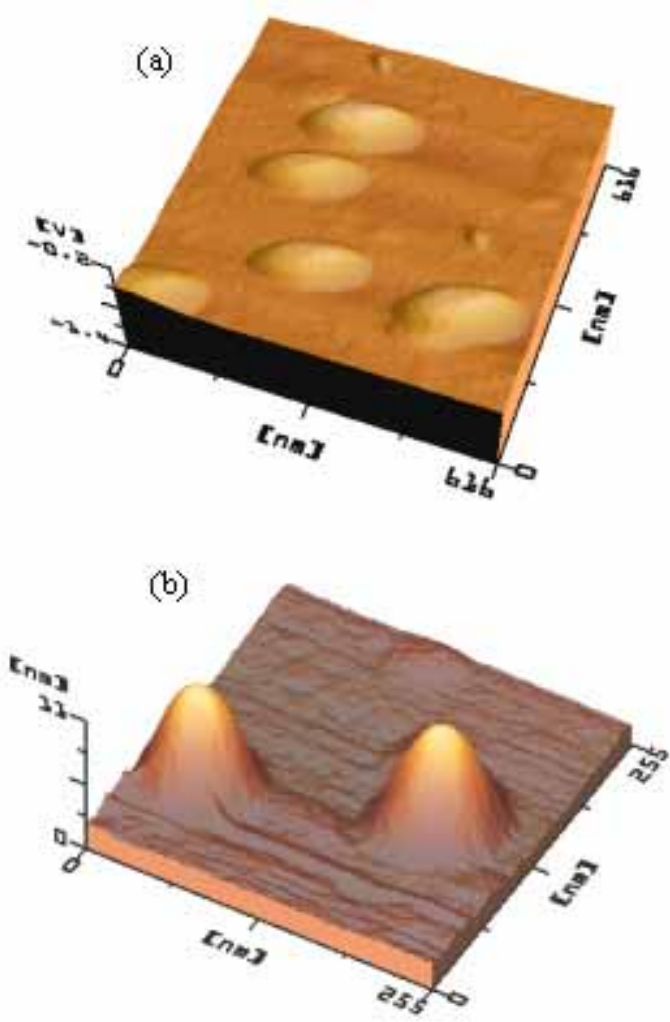

Fig. 4 3D AFM images of In islands grown on $\mathrm{Si}(100)-(2 \times 1)$ substrate at room temperature by fsPLD; (a) elongated shape, (b) hemispherical shape.

These results indicate that femtosecond PLD can produce different In island morphologies, compared to other growth techniques. The laser pulse creates transient plasma from the In target that expands rapidly in vacuum towards the $\mathrm{Si}$ substrate. Because the laser pulse terminates before the energy is completely redistributed in the target materials, the fs laser pulse tends to have a better ablation efficiency [13]. Low laser fluencies of $0.4-0.6 \mathrm{~J} / \mathrm{cm}^{2}$ were chosen to grow In films in order to limit the heating of the surrounding lattice of the In target. This results in reducing droplets, which minimize the particulate generation in the grown film. It has been shown that fs laser ablation formed fast confined plume containing energetic species [14]. The In plasma plume expands into vacuum until arriving at the Si substrate. The initial high density of the In nucleus on the surface lead to the initial development of a smooth film. Further deposition of In lead to the formation of 3D islands that form in order to accommodate the strain in the film resulting from the $\sim 15 \%$ lattice mismatch between In and $\mathrm{Si}$. Due to the pulsed nature of the PLD, the In adatoms reorganize during pulses. When the In adatoms are affected by the structure of the 2D layers, elongated 3D islands were formed. The hemispherical islands were located close to the sample edges, as noticed from the AFM images, and could be related to the preparation of the $\mathrm{Si}(100)-(2 \times 1)$ surface.

Below a substrate temperature of $350{ }^{\circ} \mathrm{C}$, the $(2 \times 1)$ structure changed to $(1 \times 1)$ during In growth, while at a substrate temperature of $350-400{ }^{\circ} \mathrm{C}$, In formed weak $(4 \times 3)$ structure. Indium grown on $\mathrm{Si}(100)-(2 \times 1)$ was observed to form In- $(4 \times 3)$ superstructure, in a layer-by-layer growth mode, when the substrate temperature was $400-420{ }^{\circ} \mathrm{C}$ and the laser fluence was $0.5 \mathrm{~J} / \mathrm{cm}^{2}$. The RHEED pattern of the In- $(4 \times 3)$ structure, Fig. 5(a), shows streaky fractional orders with sharp spots indicating the growth of smooth, high quality epitaxial In film. When In deposition was initiated, the $\mathrm{Si}(100)-(2 \times 1)$ reconstruction disappeared and the In- $(4 \times 3)$ structure formed as observed from the RHEED patterns. The $(4 \times 3)$ structure was formed only on well-prepared $\mathrm{Si}(100)-(2 \times 1)$ surface as seen from the RHEED patterns. The In- $(4 \times 3)$ structure did not form when the RHEED pattern showed high background.
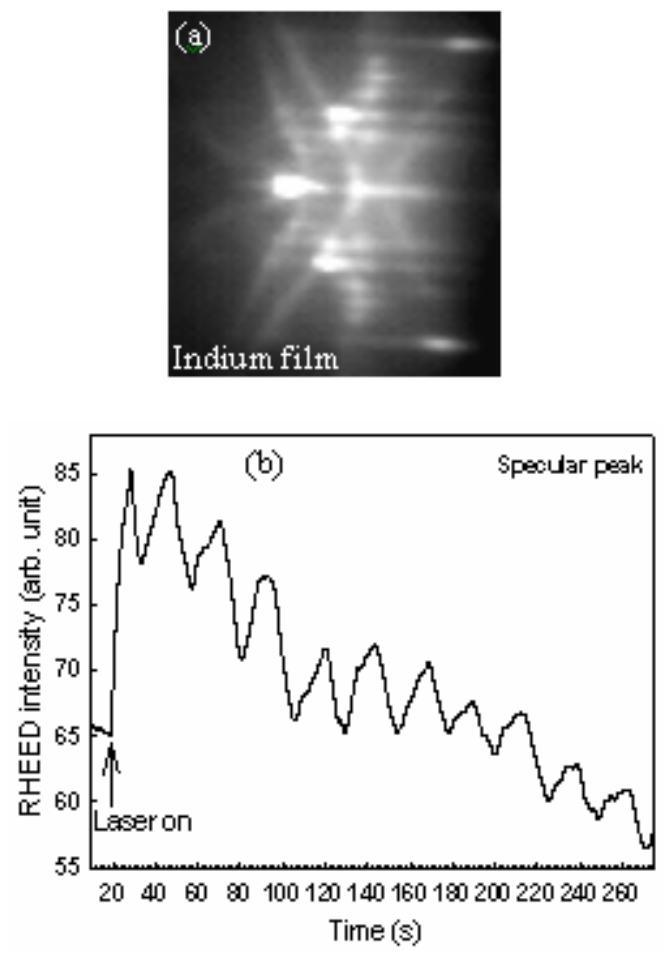

Fig. 5 (a) RHEED pattern of the grown In film on $\mathrm{Si}(100)-(2 \times 1)$ substrate at $400{ }^{\circ} \mathrm{C}$ and (b) RHEED intensity oscillations of the specular beam during growth the In- $(4 \times 3)$ structure. 
For a substrate temperature of $400{ }^{\circ} \mathrm{C}$ and a laser repetition rate of $1 \mathrm{~Hz}$, the growth mode was found to change with the laser fluence. Below a laser fluence of 0.4 $\mathrm{J} / \mathrm{cm}^{2}$ the RHEED specular intensity decreased during deposition while in the range of $0.4-0.6 \mathrm{~J} / \mathrm{cm}^{2}$ specular intensity oscillations were observed. Figure 5(b) shows RHEED oscillations of the specular beam during In growth on $\mathrm{Si}(100)-(2 \times 1)$ substrate at $400{ }^{\circ} \mathrm{C}$ (layer-by-layer mode). The oscillation period depends on the deposition rate, $\sim 0.05 \mathrm{ML} /$ pulse, and the monolayer height of the In film. Figure 6 shows AFM image of the grown In- $(4 \times 3)$ film at $400{ }^{\circ} \mathrm{C}$ after decreasing the substrate temperature to RT. Line scans show spherical shaped In nanoclusters grown on surface terraces with average size of $\sim 20 \mathrm{~nm}$. When the substrate was heated above $500{ }^{\circ} \mathrm{C}$, the $(4 \times 3)$ film disappeared and the $(2 \times 1)$ structure is appeared again as before deposition. This is due to In film evaporation. Changing the laser fluence was observed to have a similar effect to increasing the substrate temperature. This is due to the increase in adatom mobility by either increasing the substrate temperature or the adatom energy that is affected by the laser fluence.

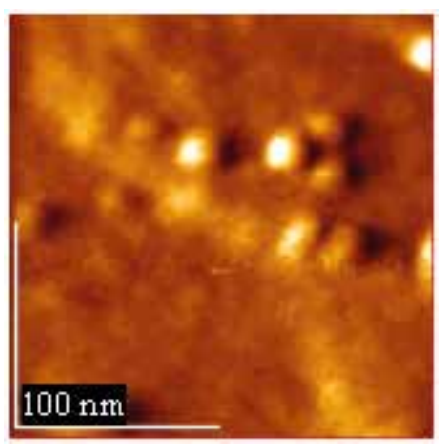

Fig. 6 AFM image of In- $(4 \times 3)$ film grown at substrate temperature of $400{ }^{\circ} \mathrm{C}$.

In conclusion, fsPLD of In on $\mathrm{Si}(100)-(2 \times 1)$ showed different film structures and morphologies. At RT In formed epitaxial 2D layers on $\mathrm{Si}(100)-(2 \times 1)$ surface followed by $3 \mathrm{D}$ islands. The In islands grew in different shapes such as elongated and hemispherical. At substrate temperatures of $400-420{ }^{\circ} \mathrm{C}$, In films initially grew in a layer-by-layer mode followed by nanoclusters formation.

\section{References:}

[1] D. B. Chrisey and G.K. Hubler: "Pulsed Laser Deposition of Thin Films”, (Wiley, New York, 1994).

[2] J. C. Miller and R. F. Haglund,: "Laser Ablation and Desorption,” Vol. 30, (Academic Press, London, 1998)

[3] M. A. Hafez, K. A. Elamrawi, and H. E. Elsayed-Ali, Appl. Surf. Sci., 233, (2004) 42.

[4] M. S. Hegazy and H. E. Elsayed-Ali, J. Vac. Sci. Tech. A, 20, (2002) 2068.

[5] D. Bauerle, Laser Processing and Chemistry, $2^{\text {nd }}$ edn. (Springer, Berlin, Heidelberg 1996)

[6] J. Knall, J.-E. Sundgren, J. E. Greene, A. Rockett, and S. A. Barnett, Appl. Phys. Lett., 45, (1984) 689.
[7] P. C. Sharma, K. W. Alt, D. Y. Yeh, and K. L. Wang, Appl. Phys. Lett., 75, (1999) 1273.

[8] B. H. Koo, T. Hanada, H. Makino, J. H. Chang, and T. Yao, J. Cryst. Growth, 229, (2001) 142.

[9] D. J.-L. Li, X.-L. Liang, J.-F. Jia, X. Liu, J.-Z. Wang, E.-G. Wang, and Q.-K. Xue, Appl. Phys. Lett., 79, (2001) 2826.

[10] J.-Z. Wang, J.-F. Jia, X. Liu, W.-D. Chen, and Q.-K. Xue, Phys. Rev. B, 65, (2002) 235303.

[11] J.-L. Li, J.-F. Jia, X.-J. Liang, X. Liu, J.-Z. Wang, Q.-K. Xue, Z.-Q. Li, J.S. Tse, Z.-Y. Zhang, and S.B. Zhang, Phys. Rev. Lett., 88, (2002) 066101.

[12] H. T. Yang and P. M. Mooney, J. Appl. Phys., 58, (1985) 1854.

[13] J. Shen, Z. Gai, and J. Kirschner, Surf. Sci. Reports, 52, (2004) 163.

[14] J. Perriere, E. Millon, W. Seiler, C. BoulmerLeborgne, V. Carciun, O. Albert, J. C. Loulergue, and J. Etchepare, J. Appl. Phys., 91, (2002) 690.

(Received: April 4, 2005, Accepted: October 17, 2005) 